\title{
An XMM-Newton view of the dipping low-mass X-ray binary XTE J1710-281
}

\author{
G. Younes ${ }^{1}$, L. Boirin ${ }^{1}$, and B. Sabra ${ }^{2}$ \\ 1 Observatoire Astronomique de Strasbourg, 11 rue de l'Université, 67000 Strasbourg, France \\ e-mail: younes@astro.u-strasbg.fr \\ 2 Department of Sciences, Notre Dame University-Louaize, PO Box 72, Zouk Mikael, Lebanon
}

Received 7 November 2008 / Accepted 24 May 2009

\section{ABSTRACT}

\begin{abstract}
Context. Studying the spectral changes during the dips exhibited by almost edge-on, low-mass X-ray binaries (LMXBs) is a powerful means of probing the structure of accretion disks. The XMM-Newton, Chandra, or Suzaku discovery of absorption lines from Fe XXV and other highly-ionized species in many dippers has revealed a highly-ionized atmosphere above the disk. A highly (but less strongly) ionized plasma is also present in the vertical structure causing the dips, together with neutral material.

Aims. We aim to investigate the spectral changes during the dips of XTE J1710-281, a still poorly studied LMXB known to exhibit bursts, dips, and eclipses.

Methods. We analyze the archived XMM-Newton observation of XTE J1710-281 performed in 2004 that covered one orbital period of the system $(3.8 \mathrm{~h})$. We modeled the spectral changes between persistent and dips in the framework of the partial covering model and the ionized absorber approach.

Results. The persistent spectrum can be fit by a power law with a photon index of $1.94 \pm 0.02$ affected by absorption from cool material with a hydrogen column density of $(0.401 \pm 0.007) \times 10^{22} \mathrm{~cm}^{-2}$. The spectral changes from persistent to deep-dipping intervals are consistent with the partial covering of the power-law emission. Twenty-six percent of the continuum is covered during shallow dipping, and $78 \%$ during deep dipping. The column density decreases from $77_{-38}^{+67} \times 10^{22} \mathrm{~cm}^{-2}$ during shallow dipping to $(14 \pm 2) \times 10^{22} \mathrm{~cm}^{-2}$ during the deep-dipping interval. We do not detect any absorption line from highly ionized species such as Fe XXV. However, the upper-limits we derive on their equivalent width (EW) are not constraining. Despite not detecting any narrow spectral signatures of a warm absorber, we show that the spectral changes are consistent with an increase in column density and a decrease in ionization state of a highly-ionized absorber, associated with an increase in column density of a neutral absorber, in agreement with the recent results found in other dippers. In XTE J1710-281, the column density of the ionized absorber increases from $4.3_{-0.5}^{+0.4} \times 10^{22} \mathrm{~cm}^{-2}$ during shallow dipping to $11.6_{-0.6}^{+0.4} \times 10^{22} \mathrm{~cm}^{-2}$ during deep dipping, while the ionization parameter decreases from $10^{2.52}$ to $10^{2.29} \mathrm{erg} \mathrm{s}^{-1} \mathrm{~cm}$. The parameters of the ionized absorber are not constrained during persistent emission. The neutral absorber only slightly increases from $(0.410 \pm 0.007) \times 10^{22} \mathrm{~cm}^{-2}$ during persistent emission to $(0.420 \pm 0.009) \times 10^{22} \mathrm{~cm}^{-2}$ during shallow dipping and to $(0.45 \pm 0.03) \times 10^{22} \mathrm{~cm}^{-2}$ during deep dipping. The warm absorber model better accounts for the $\sim 1 \mathrm{keV}$ depression visible in the pn dipping spectra, and naturally explains it as a blend of lines and edges unresolved by pn. A deeper observation of XTE J1710-281 would enable this interpretation to be confirmed.
\end{abstract}

Key words. accretion, accretion disks - stars: individual: XTE J1710-281 - X-rays: general

\section{Introduction}

XTE J1710-281 was discovered in 1998 serendipitously by RXTE at a position consistent with the location of the unidentified ROSAT source 1RXS J171012.3-280754 (Markwardt et al. 1998). Its flux varies between about 2 and $10 \mathrm{mCrab}$ on timescales of about 30 days (Markwardt et al. 2001). XTE J1710-281 shows X-ray bursts indicating that the compact object is a neutron star and that the system could lie at a distance of 15-20 kpc (Markwardt et al. 2001) or 12-16 kpc (Galloway et al. 2008). Its light curve presents eclipses lasting $410 \mathrm{~s}$ and dipping activity, both recurring at the orbital period of $3.28 \mathrm{~h}$. The dips are believed to be due to obscuration in the thickened, azimuthally structered, outer regions of an accretion disk (White \& Swank 1982). The presence of eclipses indicates that the source is viewed close to edge-on, at an inclination angle, $i$, of $75^{\circ}-80^{\circ}$ (Frank et al. 1987), where $i$ is defined as the angle between the line of sight and the rotation axis of the accretion disk. The RXTE PCA spectrum is consistent with either thermal bremsstrahlung ( $k T=14 \pm 3 \mathrm{keV}$ ) or a power law (photon index $1.8 \pm 0.1)$, with interstellar absorption $N_{\mathrm{H}}<2 \times 10^{22} \mathrm{~cm}^{-2}$ (Markwardt et al. 1998).

The 1-10 keV spectra of most of the dip sources become harder during dipping. However, these changes are inconsistent with a simple increase in photo-electric absorption by cool material. These changes have been often modeled by the "progressive covering", or "complex continuum" approach (e.g., Church et al. 1997), in which the X-ray emission is assumed to originate from a point-like blackbody, or disk-blackbody component, and from a power-law component accounting for an extended corona. This approach models the spectral changes during dipping intervals by the partial and progressive covering of the extended component by an opaque neutral absorber. The absorption of the pointlike component is allowed to vary independently from that of the extended component.

The improved sensitivity and spectral resolution of Chandra, XMM-Newton and Suzaku is allowing narrow absorption features from highly ionized $\mathrm{Fe}$ and other metals to be observed 
from a growing number of X-ray binaries. In particular, Fe XXV (He-like) or Fe XXVI (H-like) 1s-2p resonant absorption lines near $7 \mathrm{keV}$ were reported from several micro-quasars and neutron star LMXBs (e.g., Kubota et al. 2007; Sidoli et al. 2001) that are almost all viewed close to edge-on, many of them being dippers (see e.g. Table 5 of Boirin et al. 2004a). This indicates that the highly ionized plasma probably originates in an accretion disk atmosphere or wind, which could be a common feature of accreting binaries but primarily detected in systems viewed close to edge-on. During the dips, absorption lines are also detected and correspond to electronic transitions from less ionized species than during the persistent intervals. Boirin et al. (2005) demonstrated that an increase in column density and a decrease in the ionization state of a highly-ionized absorber, associated with the increase in column density of a local neutral absorber could model the changes between persistent and dipping intervals both in the X-ray continuum and the narrow absorption features of 4U 1323-62. This result was successfully applied to X 1254-690, X 1624-49, MXB 1658-298, 4U 1746-371 and XB 1916-053 observed with XMM-Newton (Díaz Trigo et al. 2006).

Here, we report on a spectral study of the archived XMMNewton observation of the dipping LMXB XTE J1710-281. We report spectral hardening from persistent to dipping intervals and model it in the framework of the partial covering appraoch and of the warm absorber approach.

\section{Observation and data reduction}

\subsection{Data reduction}

The XMM-Newton Observatory (Jansen et al. 2001) includes three $1500 \mathrm{~cm}^{2} \mathrm{X}$-ray telescopes each with an European Photon Imaging Camera (EPIC) at the focus. Two of the EPIC imaging spectrometers use MOS CCDs (Turner et al. 2001) and one uses pn CCDs (Strüder et al. 2001). Reflection Grating Spectrometers (RGS, den Herder et al. 2001) are located behind two of the telescopes while the $30-\mathrm{cm}$ optical monitor (OM) instrument has its own optical/UV telescope (Mason et al. 2001). XTE J1710-281 was observed by XMM-Newton for $13 \mathrm{ks}$ on 2004 February 22 between 09:55 and 13:16 UTC. The optical monitor instrument was operated in imaging mode with the $U$ and UVW1 filters (PSF FWHM of 1.55 and 2.0", respectively). The $U$ filter bandpass is between $\sim 300$ and $400 \mathrm{~nm}$ while the UVW1 filter covers the $\sim 230-370 \mathrm{~nm}$ wavelength range. The EPIC-pn and MOS cameras were operated in full frame mode. We concentrate here on the analysis of the EPIC-pn data since they are not affected by pile-up, contrary to MOS1 and MOS2 data where the total maximum count rate to avoid deteriorated response due to photon pile-up is $150 \mathrm{~s}^{-1}$, lot less than the total maximum count rate in the pn case, $1000 \mathrm{~s}^{-1}$. We also use RGS data. All data products were obtained from the XMM-Newton public archive and reduced using the Science Analysis System (SAS) version 8.0. Few intervals of enhanced solar activity were present. However, we did not discard any of them since the background count rate represents at maximum $3 \%$ of the source persistent rate.

We selected only single and double events (patterns 0 to 4 ) from the pn data. We extracted source events from a circle of $40^{\prime \prime}$ radius centered on the source. Background events were obtained from a circle of the same radius on the same CCD as the source but centered away. We generated response matrix files using the SAS task rmfgen, while ancillary response files were generated using the SAS task arfgen. We rebinned the EPIC-pn spectra to oversample the full-width at half maximum (FWHM) of the energy resolution by a factor 3 , and to have a minimum of 25 counts per bin to allow the use of the $\chi^{2}$ statistic.

The SAS task rgsproc was used to produce calibrated RGS event lists, spectra, and response matrices. RGS order 2 is not used in the analysis because it shows few source events detection. The RGS 1st order spectrum does not show evidence for any narrow spectral features therefore it was mainly used to check for consistency with the EPIC-pn spectra and to better constrain the low-energy part of the spectrum. It was rebinned to have a minimum of 25 counts per bin.

\subsection{Spectral analysis}

We use EPIC-pn spectra in the energy range $0.2-10 \mathrm{keV}$. We restrict the RGS spectra (RGS1 and RGS2, order 1) to the energy range of $0.5-2.0 \mathrm{keV}$ because very few source events are detected below $0.5 \mathrm{keV}$.

Spectral analysis was performed using XSPEC (Arnaud 1996) version 12.0. The updated photo-electric cross sections and the revised solar abundances of Wilms et al. (2000) ("abund wilm" command within XSPEC) are used throughout to account for absorption by neutral gas. Spectral uncertainties are given using $\Delta \chi^{2}$ of 2.71 , corresponding to $90 \%$ confidence for one interesting parameter, and to $95 \%$ confidence for upper limits. All EWs are quoted with positive values for absorption features.

\section{Results}

\subsection{Source position}

The second XMM-Newton serendipitous source catalog (2XMM) (Watson et al. 2009) gives XTE J1710-281 a position of RA $=17 \mathrm{~h} 10^{\prime} 12.532^{\prime \prime}$, Dec $=-28^{\circ} 07^{\prime} 50.95^{\prime \prime}$ (equinox 2000.0) with a 1 sigma uncertainty of $1^{\prime \prime}$ in both RA and Dec. There is no source consistent with this X-ray position in the infra-red 2MASS catalog, neither in the optical image derived from the XMM-Newton optical monitor, nor in the USNO catalog which is the deepest optical catalog that we cross-correlated with the 2XMM using the software XCAT-DB (Pineau et al. 2008).

\subsection{Lightcurve and hardness ratio}

The EPIC-pn $0.2-10 \mathrm{keV}$ source and background light curves are shown in the lower panel of Fig. 1 with a binning of $60 \mathrm{~s}$. The observation covers almost one orbital period $(3.28 \mathrm{~h})$ of the system. One eclipse lasting for $410 \mathrm{~s}$ is visible, as well as some dipping activity at the biginning and the end of the observation. The interval where the count rate remains approximately constant is referred to as the persistent emission.

We define the hardness ratio as the counts in the $2.0-10 \mathrm{keV}$ energy range (the "hard" energy band), divided by the counts in the $0.2-2.0 \mathrm{keV}$ energy range (the "soft" energy band). The hardness ratio evolution as a function of time is shown in the upper panel of Fig. 1. An increase in hardness during dipping is observed. This indicates that the number of counts in the soft band has decreased more than that in the hard band. This suggests that absorption is the main ingredient of the dipping activity. The deepest troughs of the dips are clearly associated with the strongest hardening, and hence the strongest absorption.

Based on the entire lightcurve, we extracted the spectrum of the deep-dipping state from time intervals where the count rate is less than 5 counts $\mathrm{s}^{-1}$. We extracted the shallow-dipping spectrum from time intervals with count rates between 5 and 

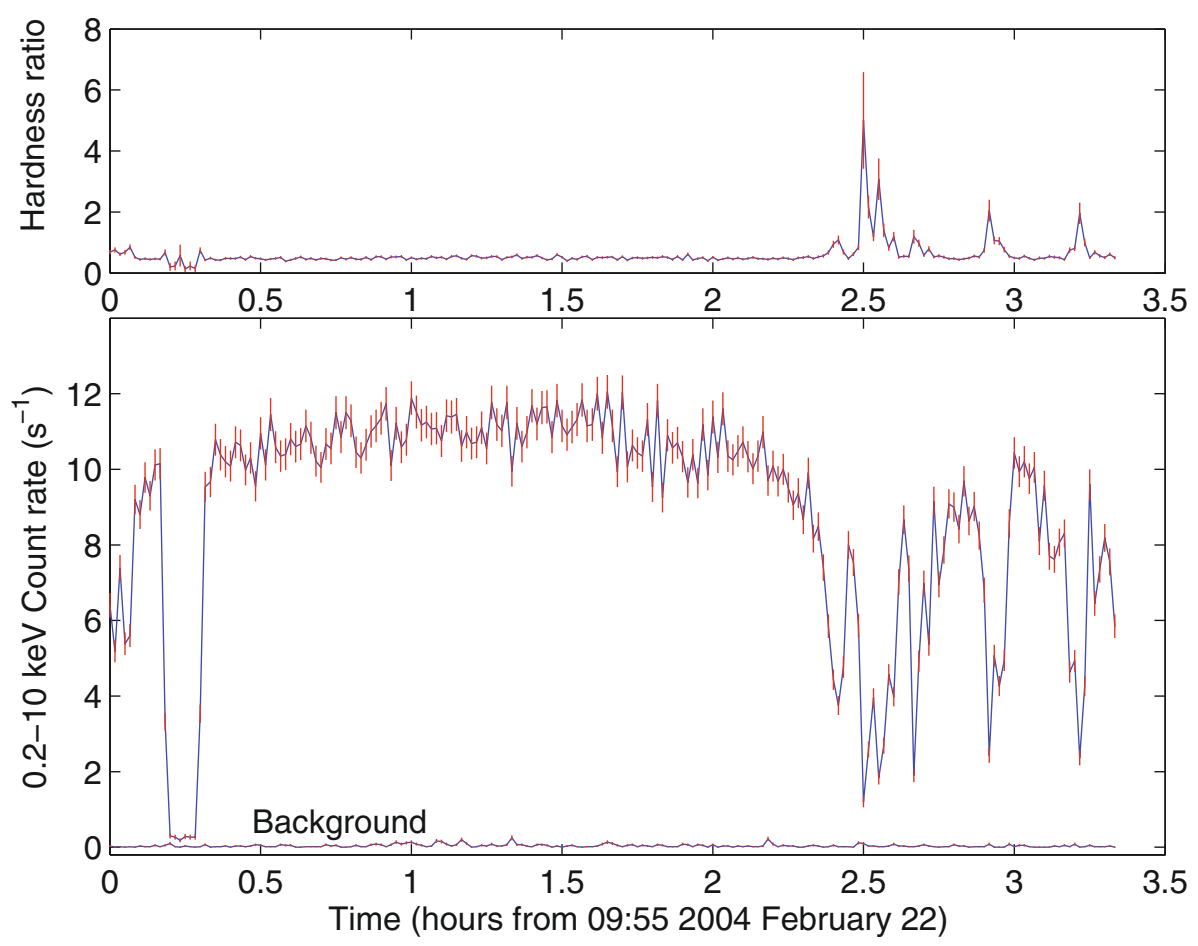

Fig. 1. The lower panel shows the $0.2-10 \mathrm{keV}$ EPIC-pn light curve of XTE J1710-281. An eclipse and the dipping activity are clearly visible. The $0.2-10 \mathrm{keV}$ background light curve is also displayed. The upper panel shows the hardness ratio (counts in the $2.0-10 \mathrm{keV}$ band divided by those between $0.2-2.0 \mathrm{keV}$ ). The dips are associated with hardening. The binning is $60 \mathrm{~s}$ in each panel.

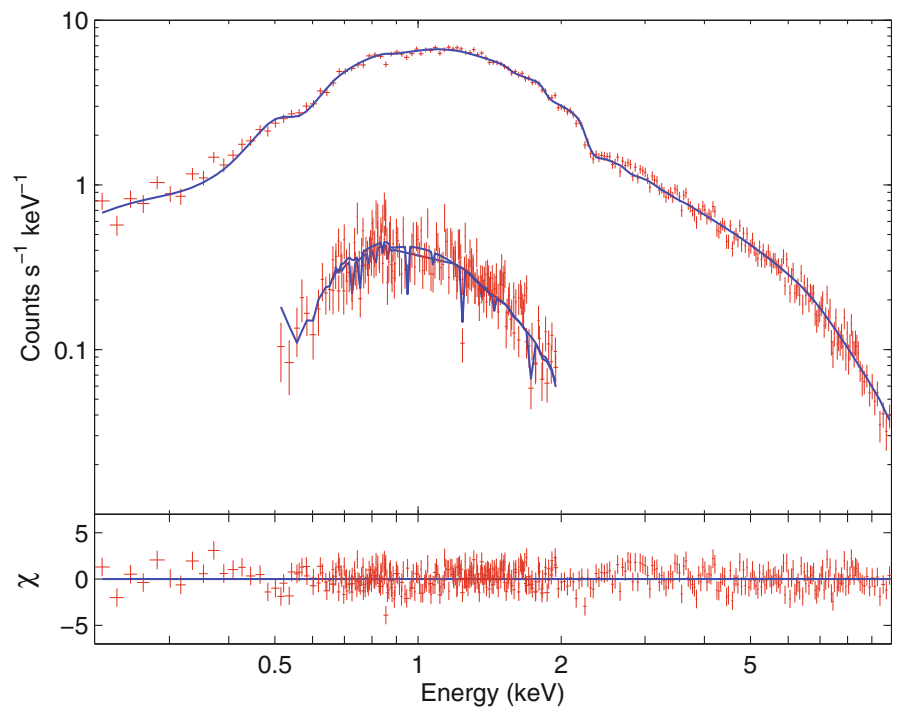

Fig. 2. The upper panel shows the EPIC pn spectrum (top), the RGS1 and RGS2 first order spectra (bottom) of XTE J1710-281 during persistent intervals. The solid line is the best-fit absorbed power-law model. The lower panel shows the spectral residuals from the best-fit model in terms of sigmas.

10 counts $\mathrm{s}^{-1}$. Finally, we extracted the spectrum of the persistent state from time intervals with count rates greater than 10 counts $\mathrm{s}^{-1}$. Of course, the eclipse interval is when the count rate approached that of the background for a total of $410 \mathrm{~s}$.

\subsection{Persistent spectrum}

We fit simultaneously the pn persistent spectrum, the RGS1 first order spectrum and the RGS2 first order spectrum. We include a multiplicative constant factor, fixed to 1 for the EPIC-pn spectrum but allowed to vary for each RGS spectrum, to take account for cross calibration uncertainties. An absorbed blackbody model or an absorbed disk-blackbody did not give acceptable fits to the persistent spectrum (reduced $\chi^{2}$ of: 14 for 430 degrees of freedom (d.o.f.) and 5 for 430 d.o.f. respectively). An absorbed power law (tbabs*pow within XSPEC) was able to fit the spectra well with a reduced $\chi^{2}$ of 1.17 for 430 d.o.f. (Fig. 2). A model consisting in two emission components, a power law and a blackbody, modified by a neutral absorber gave a reduced $\chi^{2}$ of 1.16 for 428 d.o.f. An F-test indicates that the probability for such an improvement (compared to the fit with an absorbed power law) to occur by chance is 0.16 . Therefore, the blackbody component is not significant and was not kept in the model. The best fit photon index is $1.94 \pm 0.02$ while the neutral hydrogen column density is $(0.401 \pm 0.007) \times 10^{22} \mathrm{~cm}^{-2}$. We refer hereafter to this absorption as foreground absorption, it includes contributions from the interstellar medium and/or absorption intrinsic to the system itself. The normalization of the power law is $(1.21 \pm 0.02) \times 10^{-2}$ photons $\mathrm{keV}^{-1} \mathrm{~cm}^{-2} \mathrm{~s}^{-1}$ and the constant factors are $1.11 \pm 0.05$ and $1.10 \pm 0.04$ for the RGS1 and RGS2 data respectively.

In the $0.2-10 \mathrm{keV}$ energy range, we derive an absorbed flux of $4.69 \times 10^{-11} \mathrm{erg} \mathrm{cm}^{-2} \mathrm{~s}^{-1}$, an unabsorbed flux of $7.79 \times$ $10^{-11} \mathrm{erg} \mathrm{cm}^{-2} \mathrm{~s}^{-1}$, and a luminosity of $L_{\mathrm{x}}=2.4 \times 10^{36} \mathrm{erg} \mathrm{s}^{-1}$ assuming a source distance of $16 \mathrm{kpc}$.

We do not detect absorption lines due to highly ionized elements (Fe XXV or Fe XXVI near $7 \mathrm{keV}$ ) in the persistent emission spectrum.

\subsection{Persistent, shallow and deep-dipping spectra}

We consider the dipping activity as due to the passage of a thickened part of the disk in front of the underlying X-ray source, at each orbital rotation of the system. Since the count rate and the hardness ratio of XTE J1710-281 are constant during the persistent interval (see Fig. 1), we may assume that the underlying X-ray emission does not change significantly during the whole observation, and that the spectral changes observed during the dipping interval are indeed arising from the passage of 


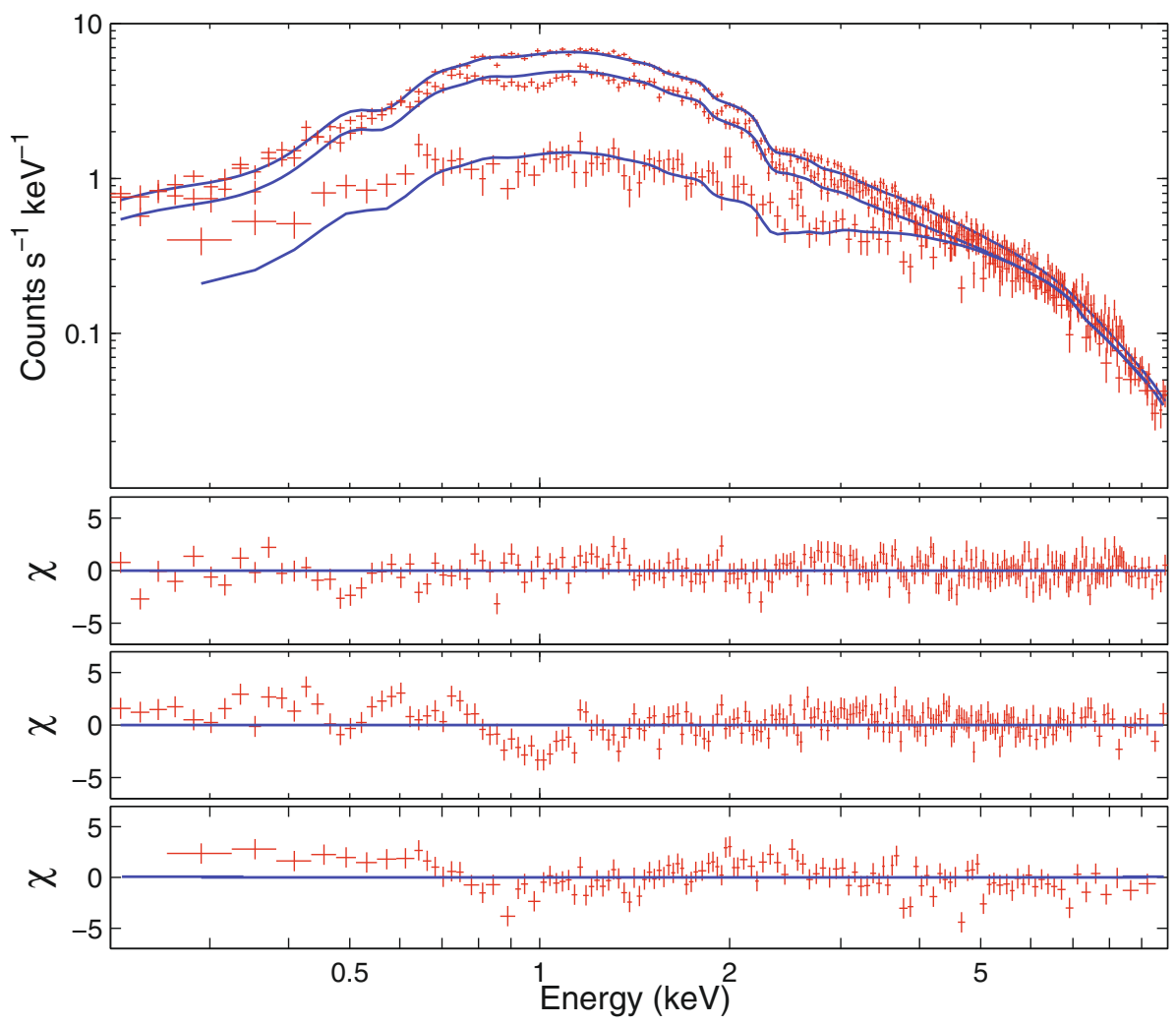

Fig. 3. The upper panel shows, from top to bottom, the EPIC pn persistent, shallow-dipping and deep-dipping spectra of XTE J1710-281, fit simultaneously with the partial covering model (see Sect. 3.4.1). The three other panels show the residuals of each spectrum from the model, in terms of sigmas. some absorbing material in the line-of-sight. The rapid variability of the spectral changes during the dipping interval indicates that this effect dominates over a potentially coincident smooth and slow spectral transition of the underlying X-ray source itself.

Therefore, to explain the spectral changes during dipping, we fit the persistent, shallow and deep-dipping spectra simultaneously, and we link the parameters of the power law component. For the persistent interval, we use pn and RGS (order 1) spectra but plot only pn for clarity. For the dipping intervals, we use only the pn spectra because too few counts are detected by RGS. In a first step, we allowed the foreground column density to be different for the three spectra, but did not obtain any acceptable fit (reduced $\chi^{2}$ of 4.4 for 769 d.o.f.). Consequently, we tested a partial covering approach (Sect. 3.4.1) and a warm absorber approach (Sect. 3.4.2).

We do not detect any absorption line from highly ionized species such as Fe XXV in XTE J1710-281, neither in the persistent spectrum nor in the dipping spectra. By including a Gaussian profile with a width fixed to 0 at $6.65 \mathrm{keV}$, the theoretical energy of the Fe XXV 1s-2p transition, we derived upperlimits of 114,50 , and $73 \mathrm{eV}$ on the $\mathrm{EW}$ of this line in the persistent, shallow-dipping, and deep-dipping spectra, respectively. Since lines from Fe XXV or other species were detected with an EW down to $25 \mathrm{eV}$ in other binaries (e.g. Boirin et al. 2005), the upper-limits derived for XTE J1710-281 are not constraining. The non detection of absorption lines in XTE J1710-281 could be explained by a lack of statistics in the current XMM-Newton observation.

\subsubsection{Progressive covering model}

In this approach, we allow the continuum emission to be partially covered by some neutral material during dipping. Since the continuum emission of XTE J1710-281 may be simply represented by a power law, we define the model of the absorbed spectrum as

$$
F(E)=\mathrm{e}^{-\sigma(E) N_{\mathrm{H}}^{\text {fore }}}\left[I_{\text {pow }}\left(f \mathrm{e}^{-\sigma(E) N_{\mathrm{H}}^{\text {pow }}}+(1-f)\right)\right]
$$

where $I_{\text {pow }}$ is the energy dependent flux of the power-law component, $N_{\mathrm{H}}^{\mathrm{pow}}$ is the column density of the absorber affecting the power law, and $N_{\mathrm{H}}^{\text {fore }}$ is the column density of the foreground absorber. The photo-electric absorption cross section, $\sigma(\mathrm{E})$, does not include Thompson scattering. The covering fraction, $f$, can vary from 0 to 1 and is unit-less.

The foreground column density, $N_{\mathrm{H}}^{\text {fore }}$, was linked for the three emission intervals assuming that the X-ray source is subject to the same foreground absorption. We fixed the covering fraction, $f$, and $N_{\mathrm{H}}^{\mathrm{pow}}$ to zero during the persistent emission, since this emission interval is not supposed to undergo any absorption from the bulge. These two parameters are allowed to vary for the shallow-dipping and the deep-dipping emission.

The spectra and best fit model are shown in Fig. 3. Table 1 gives the best fit parameter values. The reduced $\chi^{2}$ of the best fit is 1.55 for 767 d.o.f. The foreground column density is $0.38 \times$ $10^{22} \mathrm{~cm}^{-2}$, the photon index is 1.91 and the normalization of the power law is $1.17 \times 10^{-2}$ photons $\mathrm{keV}^{-1} \mathrm{~cm}^{-2} \mathrm{~s}^{-1}$. These values are consistent with those found for the persistent emission alone (Sect. 3.3). The covering fraction increases from $26 \%$ during the shallow-dipping, to $78 \%$ during the deep-dipping interval, which represents a change at a level of more than $10 \sigma$. This would indicate that the emission region is angularly more extended than the absorbing region, and that a larger fraction of it is covered during deep dipping. However, we find that $N_{\mathrm{H}}^{\mathrm{pow}}$ decreases from $77_{-38}^{+67} \mathrm{~cm}^{-2}$ during the shallow dip to $14 \pm 2 \mathrm{~cm}^{-2}$ during the deep dip interval, which represents a change at a level of $2.7 \sigma$. This would mean that the absorbing layer is thiner or less dense during deep dipping than during shallow dipping. 
Table 1. Best fit spectral parameters for the persistent, the shallow-dipping, and the deep-dipping spectra fit simultaneously with the partial covering model.

\begin{tabular}{|c|c|c|c|c|}
\hline Parameter & Per. emission & Shallow dip & & Deep dip \\
\hline$N_{\mathrm{H}}^{\text {fore }}\left(10^{22} \mathrm{~cm}^{-2}\right)$ & & $0.385 \pm 0.007$ & & \\
\hline Photon index & & $1.91 \pm 0.02$ & & \\
\hline Norm. (photons $\mathrm{keV}^{-1} \mathrm{~cm}^{-2} \mathrm{~s}^{-1}$ ) & & $0.0117 \pm 0.0002$ & & \\
\hline$N_{\mathrm{H}}^{\mathrm{pow}}\left(10^{22} \mathrm{~cm}^{-2}\right)$ & 0.0 Fixed & $77_{-38}^{+67}$ & & $14 \pm 2$ \\
\hline Significance & & & $2.7 \sigma$ & \\
\hline$f$ & 0.0 Fixed & $0.26 \pm 0.01$ & & $0.776 \pm 0.009$ \\
\hline Significance & & & $>10 \sigma$ & \\
\hline
\end{tabular}

Note: The foreground hydrogen column density, $N_{\mathrm{H}}^{\text {fore }}$, the photon index and the normalization of the power law are linked between persistent, shallow and deep-dipping emission. The covering fraction $f$ and the hydrogen column density, $N_{\mathrm{H}}^{\mathrm{pow}}$, are allowed to vary to explain the spectral changes in the spectra. Uncertainties are given at a $90 \%$ confidence level. We also indicate the significance of the change of $N_{\mathrm{H}}^{\text {pow }}$ and $f$ between shallow and deep-dipping spectra.

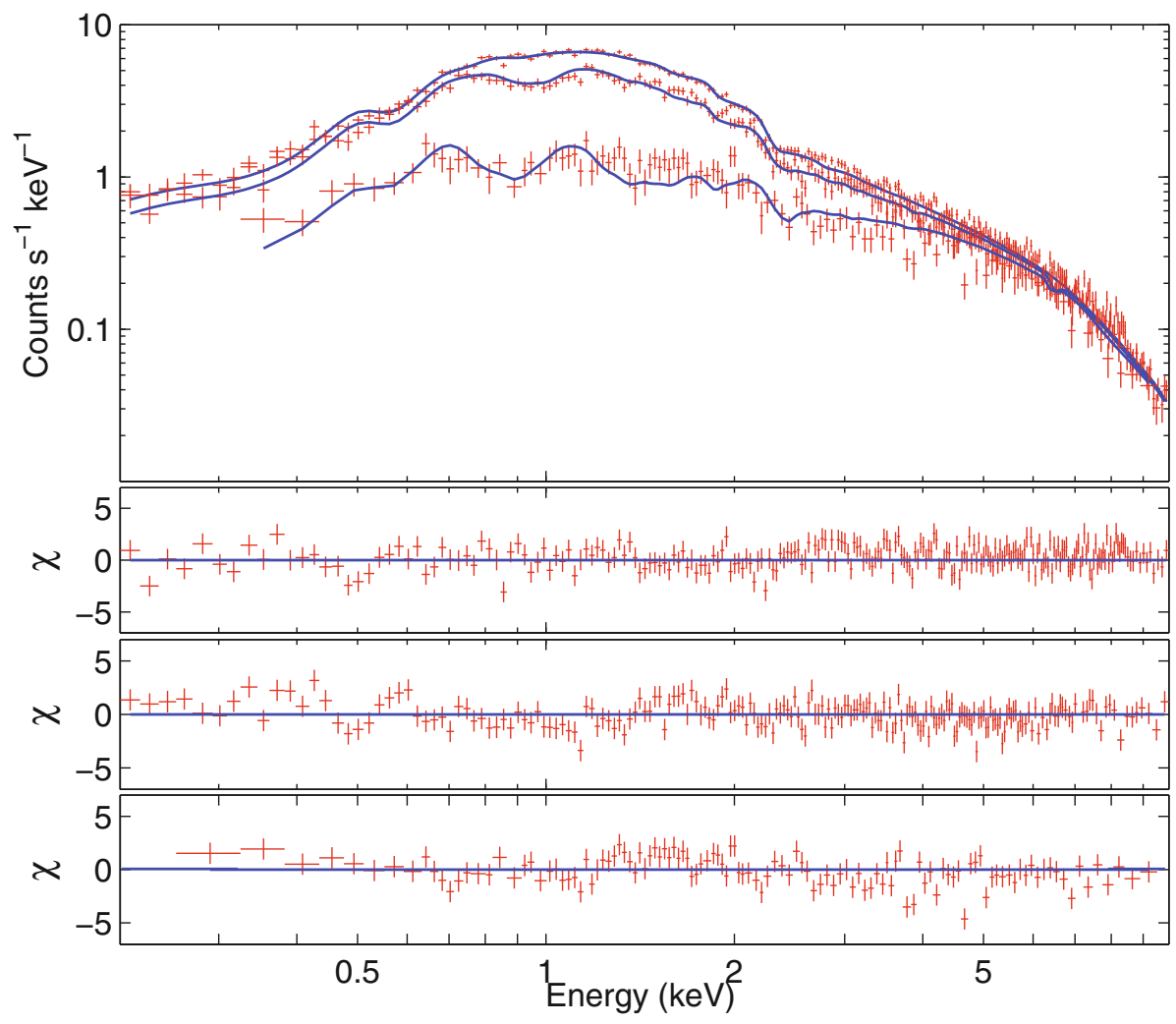

Fig. 4. The upper panel shows, from top to bottom, the EPIC pn persistent, shallow-dipping and deep-dipping spectra of XTE J1710-281, fit simultaneously with the warm absorber model (see Sect. 3.4.2). The three other panels show the residuals of each spectrum from the model, in terms of sigmas.

\subsubsection{Ionized absorber model}

As an alternative, we tested the hypothesis that the spectral changes during dipping could be due to changes in the properties of both a neutral and an ionized absorber. We used the photoionization code CLOUDY version 07.02 (Ferland et al. 1998) to simulate ionized clouds standing in the line of sight toward the $\mathrm{X}$-ray emission. Cloudy requires specifying the hydrogen density $\left(10^{8} \mathrm{~cm}^{-3}\right)$, an abundance table (Anders \& Grevesse 1989), the hydrogen column density (the hydrogen density integrated along the line of sight), an ionizing continuum, and the ionization parameter defined by Tarter et al. (1969) as

$\xi=\frac{L_{\text {ion }}}{n_{\mathrm{H}} \times r^{2}} \operatorname{erg~s}^{-1} \mathrm{~cm}$ where $n_{\mathrm{H}}$ is the hydrogen density at the illuminated face of the ionized cloud and $r$ is the source cloud separation. $L_{\text {ion }}$ is the luminosity between 1 Ryd and 1000 Ryd.

As ionizing continuum, we assumed a power law with an $\mathrm{X}$-ray slope consistent with what we determined from the persistent emission. We calculate a grid of warm absorbers each having a distinct column density/ionization parameter combination. For a fixed hydrogen density, the ionization parameter is basically a measure of the overall strength of the ionizing continuum and consequently, it affects the presence/absence of the different ionic species. On the other hand, the hydrogen column density controls the amount of the ionic species present. Together these two quantities control the amount of absorption, at every energy, 
Table 2. Best fit spectral parameters for the persistent, the shallow-dipping, and the deep-dipping spectra fit simultaneously with the ionized absorber model.

\begin{tabular}{|c|c|c|c|c|}
\hline Parameter & Per. emission & Shallow dip & & Deep dip \\
\hline \multirow[t]{2}{*}{$N_{\mathrm{H}}^{\text {fore }}\left(10^{22} \mathrm{~cm}^{-2}\right)$} & $0.410 \pm 0.007$ & $0.420 \pm 0.009$ & & $0.45 \pm 0.03$ \\
\hline & $1.4 \sigma$ & & $1.7 \sigma$ & \\
\hline Photon index & & $1.98 \pm 0.01$ & & \\
\hline Norm. (photons $\mathrm{keV}^{-1} \mathrm{~cm}^{-2} \mathrm{~s}^{-1}$ ) & & $0.0124 \pm 0.0002$ & & \\
\hline $\log (\xi)\left(\mathrm{erg} \mathrm{s}^{-1} \mathrm{~cm}\right)$ & 5.0 Frozen & $2.52_{-0.07}^{+0.05}$ & & $2.29 \pm 0.02$ \\
\hline Significance & & & $5 \sigma$ & \\
\hline$N_{\mathrm{H}}^{\xi}\left(10^{22} \mathrm{~cm}^{-2}\right)$ & 0.32 Frozen & $4.3_{-0.5}^{+0.4}$ & & $11.6_{-0.6}^{+0.4}$ \\
\hline Significance & & & $>10 \sigma$ & \\
\hline & Reduced $\chi^{2}=1.42$ for & d.o.f. & & \\
\hline
\end{tabular}

Note: The photon index and the normalization of the power law are linked between persistent, shallow and deep dipping. $N_{\mathrm{H}}^{\mathrm{fore}}$ is the foreground neutral hydrogen column density. $\xi$ is the ionization parameter. $N_{\mathrm{H}}^{\xi}$ is the warm absorber hydrogen column density. These parameters are allowed to vary to explain the spectral changes from persistent to deep dipping. Uncertainties are given at a $90 \%$ confidence level. We also indicate the significance of the change of $N_{\mathrm{H}}^{\text {fore }}$ between the three spectra as well as the significance of the change of $\log (\xi)$ and $N_{\mathrm{H}}^{\xi}$ between shallow and deep-dipping spectra.

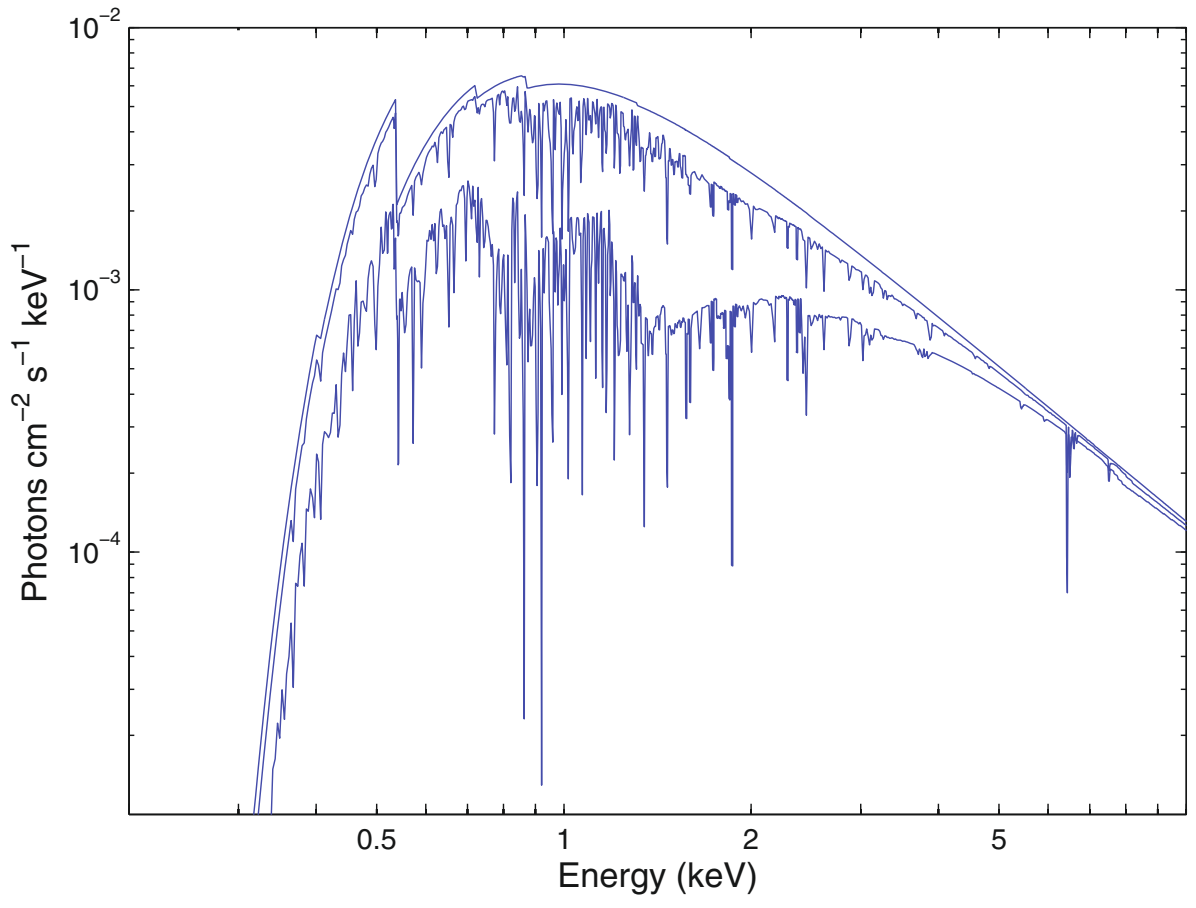

Fig. 5. The warm absorber model that fit the persistent (top), shallow-dipping (middle) and deep-dipping (bottom) spectra of XTE J1710-281 (see Sect. 3.4.2). More absorption lines and edges are present during deep dipping, when the ionization level is lower. suffered by the incident continuum upon exiting the warm absorber. The transmitted continuum bears the spectral signatures, such as absorption edges and lines, of the ions present in the warm absorber. XSPEC interpolates over the transmitted continua from the warm absorber grid to find the best fit to a spectrum.

To test the hypothesis that the spectral changes during dipping could be due to changes in the properties of both a neutral and an ionized absorber, we kept the parameters of the power law linked between persistent, shallow-dipping and deepdipping spectra, but left the parameters of the foreground absorber and of the ionized absorber free. However, since the persistent spectrum is consistent with a power law and does not show any absorption features due to highly ionized elements (see Sect. 3.3), the properties of the warm absorber, if present during persistent emission, cannot be constrained from this observation. Consequently, for the persistent spectrum, we fixed the warm absorber parameters to values such that the plasma is transparent at all energies, as if it were absent. In practise, we fixed the ionized column density to $10^{21.5} \mathrm{~cm}^{-2}$ and the ionization parameter to $10^{5.0} \mathrm{erg} \mathrm{s}^{-1} \mathrm{~cm}$, which indeed corresponds to a thin nearly fully ionized and hence transparent medium.

Figure 4 shows the spectra and best fit model and Fig. 5 shows the unfolded spectra. Table 2 gives the values of the fit parameters. The values of the photon index and the normalization of the power-law component were 1.98 and $1.2 \times$ $10^{-2}$ photons $\mathrm{keV}^{-1} \mathrm{~cm}^{-2} \mathrm{~s}^{-1}$ respectively. They are consistent with the values obtained when fitting the persistent spectra 


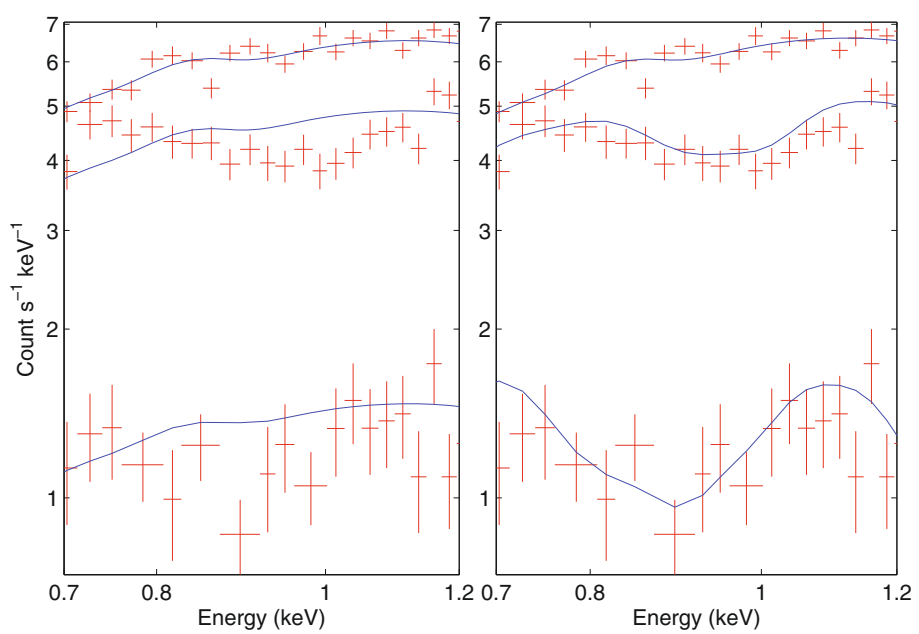

Fig. 6. A zoom at the $1 \mathrm{keV}$ region of the persistent, shallow-dipping and deep-dipping spectra fitted with either, the partial covering model (left panel) or the ionized absorber approach (right panel). The warm absorber model fits better the $1 \mathrm{keV}$ region of the dipping spectra.

alone. The foreground neutral absorption increased slightly from $(0.410 \pm 0.007) \times 10^{22} \mathrm{~cm}^{-2}$ during persistent interval to $(0.420 \pm 0.009) \times 10^{22} \mathrm{~cm}^{-2}$ during shallow dipping and to $(0.45 \pm 0.03) \times 10^{22}$ during deep dipping, which represents, between each two consecutive intervals, a change at a level of $1.4 \sigma$ and $1.7 \sigma$ respectively. The ionization parameter decreases from $10^{2.52} \mathrm{erg} \mathrm{s}^{-1} \mathrm{~cm}$ during the shallow-dipping interval to $10^{2.29} \mathrm{erg} \mathrm{s}^{-1} \mathrm{~cm}$ during the deep-dipping emission, which represents a change at a level of $5 \sigma$. The column density of the ionized absorber increases from $4.3_{-0.5}^{+0.4} \times 10^{22} \mathrm{~cm}^{-2}$ during the shallow dipping to $11.6_{-0.6}^{+0.4} \times 10^{22} \mathrm{~cm}^{-2}$ during the deep-dipping interval, which represents a change at a level of more than $10 \sigma$. These results indicate that the spectral changes during dipping in XTE J1710-281 are consistent with an increase in column density and a decrease in ionization state of a highly ionized absorber, associated with a slight increase in column density of a local neutral absorber.

\subsection{Model comparison near $\sim 1 \mathrm{keV}$}

Although the partial covering model (Sect. 3.4.1) and the warm absorber model (Sect. 3.4.2) can both fit the spectra of XTE J1710-281, we note that the warm absorber model gives a better fit, especially near $\sim 1 \mathrm{keV}$. Figure 6 shows a zoom at $1 \mathrm{keV}$ of the XTE J1710-281 spectra fitted with the ionized absorber (right panel) and the partial covering model (left panel).

The modulation of the shallow and deep-dipping spectra, near $1 \mathrm{keV}$ and $0.9 \mathrm{keV}$, respectively, is clearly better accounted for by the warm absorber model. Restricting the energy range to $0.7-1.2 \mathrm{keV}$, the reduced chi squared would be 0.99 (50 d.o.f) for the warm absorber and 1.84 (52 d.o.f.) for the partial covering model. This strongly favors the warm absorber interpretation. Indeed a "depression" such as seen in the dipping spectra near $1 \mathrm{keV}$ is most easily explained by invoking absorption than by a combination of emission components. Now, absorption by cool material does not predict strong edges near $1 \mathrm{keV}$ that would be consistent with the rest of the spectral shape. On the contrary, absorption by a warm absorber naturally includes many lines and edges from ionized species (see Fig. 5). The convolution of these narrow features with an instrument response with moderate energy resolution naturally translates into a depression such as observed in the pn dipping spectra of XTE J1710-281.

\section{Discussion}

We presented a detailed X-ray light curve covering one orbital period for the LMXB XTE J1710-281. It shows irregular dipping activity and a total eclipse, implying that the system is viewed with a high inclination angle. We fitted the persistent spectrum with an absorbed power law with a photon index of $1.94 \pm 0.02$ while the foreground hydrogen column density was $(0.401 \pm 0.007) \times 10^{22} \mathrm{~cm}^{-2}$. We did not detect absorption lines from highly ionized species such as Fe XXV resonant line at $6.65 \mathrm{keV}$ seen in other dippers, but the upper limits we derived on their EW are not constraining. The overall $0.2-10 \mathrm{keV}$ spectrum becomes harder during the dips, but this change is inconsistent with a simple increase in absorption by cool material. We modeled the spectral changes between persistent and dipping intervals in two ways.

In a first place, we modeled the spectral changes during dipping by the partial covering of the power-law emission. The covering fraction increased from shallow-dipping to the deepdipping interval. However, the neutral column density of the covering component decreased from shallow dipping to deep dipping. The increase of the covering fraction is consistent with the results obtained for other dippers studied with the partial covering model in the past (e.g. Church \& Balucinska-Church 1995). However, the decrease in column density is inconsistent with what is commonly found in LMXBs, where the column density also increases as we go deeper in the dips (e.g. Church et al. 1998; Smale et al. 2002).

In a second place, we modeled the spectral changes during dipping by the variation in the properties of an ionized absorber and of a neutral absorber. The foreground column density increases slightly from $(0.410 \pm 0.007) \times 10^{22} \mathrm{~cm}^{-2}$ during the persistent emission to $(0.45 \pm 0.03) \times 10^{22} \mathrm{~cm}^{-2}$ during deep dipping. The ionization parameter decreases from $10^{2.52} \mathrm{erg} \mathrm{s}^{-1} \mathrm{~cm}$ during the shallow-dipping interval to $10^{2.29} \mathrm{erg} \mathrm{s}^{-1} \mathrm{~cm}$ during deep dipping. On the contrary, the column density of the ionized absorber increases from $4.3_{-0.5}^{+0.4} \times 10^{22} \mathrm{~cm}^{-2}$ to $11.6_{-0.6}^{+0.4} \times 10^{22} \mathrm{~cm}^{-2}$. The properties of the ionized and neutral absorbers that we derived for XTE J1710-281, and their change during dipping, are similar to those found in the other dippers observed with XMMNewton (Boirin et al. 2005; Díaz Trigo et al. 2006), especially to those of MXB 1658-298. In this source, the ionization parameter decreases to reach $10^{2.42} \mathrm{erg} \mathrm{s}^{-1} \mathrm{~cm}$ during the deepest dips while the ionized absorber column density increases to $53 \times 10^{22} \mathrm{~cm}^{-2}$. We note that the change in the foreground column density, $N_{\mathrm{H}}^{\text {fore }}$, between persistent and the deepest dips in XTE J1710-281 is very small compared to MXB 1658-298. This could partially be due to the fact that we split the dips into two dipping levels while the dips in MXB 1658-298 were split into five levels. Additionally, Díaz Trigo et al. (2006) were able to explain the spectral changes in the $0.2-10 \mathrm{keV}$ continuum with the change in the properties of a warm absorber and a neutral one from 4U 1746-371 and EXO 0748-676, the two other sources that do not show any spectral signatures due to highly ionized species near $7 \mathrm{keV}$.

Although the partial covering model and the warm absorber model can both fit the spectra of XTE J1710-281, the depression in the dipping spectra near $1 \mathrm{keV}$ is clearly better accounted for by the warm absorber model. It is naturally explained by the many lines and edges produced by the ionized absorber 
(and not by the neutral one), but not resolved by the EPIC-pn instrument.

Similar depressions near $1 \mathrm{keV}$ were detected in the pn spectra of several other dippers (XB 1916-053, EXO 0748-676, X 1254-690 and MXB 1658-298) and interpreted in the same way (Díaz Trigo et al. 2006). However, the signal to noise ratio of the RGS spectra acquired simultaneously for these sources was often too low, especially during dipping, to enable to confirm the interpretation by resolving the individual lines or edges that would constitute the depression seen in pn spectra. Among the dippers studied by Díaz Trigo et al. (2006), only MXB 1658-298 had shown clear absorption lines in the RGS (Sidoli et al. 2001), but this was during persistent emission, while too few counts were detected by RGS during dipping. We note however, that, before the use of warm absorber models, Boirin et al. (2004b) modeled a feature appearing in the pn spectra of XB 1916-053 with an edge at an energy decreasing from $0.98 \pm 0.02 \mathrm{keV}$ to $0.87_{-0.04}^{+0.06} \mathrm{keV}$ from persistent to deep dipping, and interpreted it as resulting from edges of moderately ionized $\mathrm{Ne}$ and/or Fe, with the average ionization state decreasing. The RGS spectra could also be accounted for by including an edge at an energy lower during dipping than during persistent intervals, consistently with pn results.

The absorption feature near $1 \mathrm{keV}$ in the dipping spectra of XTE J1710-281, together with the fact that warm absorbers were unumbigously identified thanks to the detection of some of their narrow spectral signatures in many dipping sources whose spectral changes used to be modeled by partial covering, strongly favor the warm absorber approach. This interpretation would be easily confirmed by a longer XMM observation of XTE J1710-281 that would enable the dectection of, e.g., the Fe XXV absorption line at $6.65 \mathrm{keV}$ down to an EW of about $15 \mathrm{eV}$.

\section{References}

Anders, E., \& Grevesse, N. 1989, Geochim. Cosmochim. Acta, 53, 197

Arnaud, K. A. 1996, in Astronomical Data Analysis Software and Systems V, ASP Conf. Ser., 101, 17

Boirin, L., Parmar, A. N., Barret, D., Paltani, S., \& Grindlay, J. E. 2004a, A\&A, 418, 1061

Boirin, L., Parmar, A. N., Barret, D., Paltani, S., \& Grindlay, J. E. 2004b, A\&A, 418, 1061

Boirin, L., Méndez, M., Díaz Trigo, M., Parmar, A. N., \& Kaastra, J. S. 2005, A\&A, 436, 195

Church, M. J., \& Balucinska-Church, M. 1995, A\&A, 300, 441

Church, M. J., Dotani, T., Balucinska-Church, M., et al. 1997, ApJ, 491, 388

Church, M. J., Balucinska-Church, M., Dotani, T., \& Asai, K. 1998, ApJ, 504, 516

den Herder, J. W., Brinkman, A. C., Kahn, S. M., et al. 2001, A\&A, 365, L7

Díaz Trigo, M., Parmar, A. N., Boirin, L., Méndez, M., \& Kaastra, J. S. 2006, A\&A, 445, 179

Ferland, G. J., Korista, K. T., Verner, D. A., et al. 1998, PASP, 110, 761

Frank, J., King, A. R., \& Lasota, J. P. 1987, A\&A, 178, 137

Galloway, D. K., Muno, M. P., Hartman, J. M., et al. 2008, ApJS, 179, 360

Jansen, F., Lumb, D., Altieri, B., et al. 2001, A\&A, 365, L1

Kubota, A., Dotani, T., Cottam, J., et al. 2007, PASJ, 59, 185

Markwardt, C. B., Marshall, F. E., Swank, J., \& Takeshima, T. 1998, IAU Circ., 6998, 2

Markwardt, C. B., Swank, J. H., \& Strohmayer, T. E. 2001, in BAAS, 33, 1350

Mason, K. O., Breeveld, A., Much, R., et al. 2001, A\&A, 365, L36

Pineau, F.-X., Derriere, S., Michel, L., \& Motch, C. 2008, in Astronomical Data Analysis Software and Systems XVII, ed. R. W. Argyle, P. S. Bunclark, \& J. R. Lewis, ASP Conf. Ser., 394, 369

Sidoli, L., Oosterbroek, T., Parmar, A. N., Lumb, D., \& Erd, C. 2001, A\&A, 379, 540

Smale, A. P., Church, M. J., \& Bałucińska-Church, M. 2002, ApJ, 581, 1286

Strüder, L., Briel, U., Dennerl, K., et al. 2001, A\&A, 365, L18

Tarter, C. B., Tucker, W. H., \& Salpeter, E. E. 1969, ApJ, 156, 943

Turner, M. J. L., Abbey, A., Arnaud, M., et al. 2001, A\&A, 365, L27

Watson, M. G., Schröder, A. C., Fyfe, D., et al. 2009, A\&A, 493, 339

White, N. E., \& Swank, J. H. 1982, ApJ, 253, L61

Wilms, J., Allen, A., \& McCray, R. 2000, ApJ, 542, 914 